\title{
Circuit
}

Musiques contemporaines

\section{Entrevue avec Jean-François Denis, directeur d'Empreintes DIGITALes}

\section{Yves Daoust}

Volume 13, numéro 1, 2002

L’électroacoustique : à la croisée des chemins?

URI : https://id.erudit.org/iderudit/902266ar

DOI : https://doi.org/10.7202/902266ar

Aller au sommaire du numéro

Éditeur(s)

Les Presses de l'Université de Montréal

ISSN

1183-1693 (imprimé)

1488-9692 (numérique)

Découvrir la revue

Citer ce document

Daoust, Y. (2002). Entrevue avec Jean-François Denis, directeur d'Empreintes DIGITALes. Circuit, 13(1), 57-64. https://doi.org/10.7202/902266ar

\section{Résumé de l'article}

C'est à l'été de 1989 que Jean-François Denis, en collaboration avec Claude Schryer, décidait de fonder une maison de disques spécialisée en électroacoustique. Ainsi naquit Empreintes DIGITALes, aujourd'hui considérée comme l'une des étiquettes les plus importantes au monde dans le domaine. Jean-François Denis fait le point sur l'évolution de sa compagnie. d'utilisation que vous pouvez consulter en ligne.

https://apropos.erudit.org/fr/usagers/politique-dutilisation/ 


\section{Entrevue avec Jean-François Denis, directeur d'Empreintes DIGITALes}

C'est à l'été de 1989 que Jean-François Denis, en collaboration avec Claude Schryer, décidait de fonder une maison de disques spécialisée en électroacoustique. Ainsi naquit Empreintes DIGITALes, aujourd'hui considérée comme l'une des étiquettes les plus importantes au monde dans le domaine.

Jean-François Denis fait le point sur l'évolution de sa compagnie.

(Propos recueillis le 22 juin 2002 par Yves Daoust)

Yves Daoust - Qu'est-ce qui t'a motivé à mettre sur pied une maison d'enregistrement?

Jean-François Denis - À l'université Concordia, où j'enseignais à l'époque, nous produisions beaucoup de concerts, si bien que nous avions accumulé un nombre impressionnant d'œuvres sur bande qui dormaient sur les tablettes. Je me suis dit qu'il fallait que ces musiques vivent, qu'elles circulent. L'idée m'est alors venue de créer une série d'enregistrements électroacoustiques qui rendraient ces œuvres accessibles à un public plus vaste que celui du concert, qui assureraient la pérennité des œuvres. Je n'ai pas eu immédiatement l'idée de fonder ma propre compagnie. J'ai d'abord fait le tour des maisons existantes au Québec et dans l'ensemble du Canada, en leur proposant, sans succès, de développer une souscollection électroacoustique. Devant leur refus, j'ai décidé de plonger et, avec Claude Schryer, nous avons fondé notre propre maison de production lque je dirige seul depuis 1992).

Yves Daoust - C'est donc parti de l'intention de faire connaître les œuvres québécoises?

Jean-François Denis - $\|$ est vrai que ça a démarré avec des compositeurs qui m'étaient proches géographiquement. Mais rapidement le cercle s'est élargi : dès 
la deuxième année, des compositeurs étrangers, dont Denis Smalley, s'ajoutaient au catalogue. (Aujourd'hui, sur les 66 CD d'Empreintes DIGITALes, 26 sont de compositeurs étrangers...)

\section{Yves Daoust - Quel est le créneau d'Empreintes DIGITAles?}

Jean-François Denis - J'ai décidé de me limiter uniquement à l'électroacoustique, d'autres compagnies s'occupant des musiques instrumentales contemporaines. Par contre, j'avais une définition très large de l'électroacoustique: j'ai produit des œuvres mixtes (instruments acoustiques et bande), des documentaires sonores...

Yves Daoust - Donc on ratissait large?

Jean-François Denis - Oui, au début. Mais il y a quelques années, j'ai ressenti le besoin de resserrer le créneau, de me limiter uniquement, ou presque (il y a toujours des exceptions), aux musiques de support.

Yves Daoust - Intéressant... On en reparlera. Mais revenons aux débuts. Produire des disques compacts, c'est relativement facile. Mais le problème majeur n'estil pas celui de la diffusion?

Jean-François Denis - Oui, tout à fait. Le travail commence vraiment le jour où l'on vous livre les boîtes de compacts... C'est d'autant plus difficile et exigeant qu'il s'agit d'un créneau très spécialisé.

Premièrement, i'ai établi une formule claire : chaque $C D$ serait consacré à un seul compositeur, ce qui permet de personnaliser chaque album beaucoup plus que lorsqu'il s'agit d'une compilation d'œuvres de compositeurs différents. Sur le plan iconographique, i'ai fait en sorte que l'identification de l'étiquette soit claire et forte, porteuse de signification. II fallait s'assurer que le public identifie, dès le premier regard, l'étiquette à la musique électroacoustique. Quant à la distribution comme telle, sans laisser de côté le créneau traditionnel des magasins de disques, je l'ai axée davantage sur la vente par correspondance.

Yves Daoust - Puis il y eut Internet...

Jean-François Denis - Oui, bien sûr : très tôt après l'établissement de la norme du web, nous avions notre site (dès février 1995). Actuellement, je dirais que plus de $60 \%$ de nos ventes se font directement par le site Internet www.electrocd.com.

Yves Daoust - Ce qui nous amène sur le terrain du virtuel : est-il encore nécessaire aujourd'hui de produire des disques? Quand on pense aux mp3...

Jean-François Denis - Effectivement, on a accès aujourd'hui à tous les genres de musique, en les téléchargeant directement à partir, par exemple, de sites comme www.mp3.com et ce, gratuitement, sans compter que la qualité sonore est intéressante (mais elle n'égale toutefois pas le spectre et la dynamique du CD). Dans ce cas, il n'y a donc pas de producteur, mais simplement un site d'accueil où n'importe qui peut déposer sa musique. Mais estce que ça fonctionne? D'une part, on est 
privé d'iconographie : un disque, c'est un objet qui peut être beau, original dans sa présentation; et puis il y a le livret qui est très précieux, particulièrement dans le cas de musiques de recherche et de création. D'autre part, on est face à une quantité étourdissante d'information, dans toutes les directions musicales. Comment choisir?

Yves Daoust - Oui, mais qu'est-ce que je risque à part une perte de temps? C'est gratuit, et puis ça permet de faire des découvertes, d'élargir ses horizons...

Jean-François Denis - Sûrement, si tu es prêt à y consacrer l'essentiel de ton temps. Mais je continue de croire que le rôle du producteur demeure primordial. Non seulement il assure le suivi et fait la promotion des titres qu'il produit, mais il donne une couleur, une orientation artistique qui permet, encore une fois, une identification claire du genre par le public et l'établissement d'une relation de confiance avec lui : on connaît le type de produits d'une étiquette donnée. Et si on aime, on sera porté à faire confiance au producteur et à se procurer un nouvel album, même si on ne connaît pas le compositeur. II me semble très important de garder ce principe de parrainage, de direction artistique.

Yves Daoust - Donc tu ne crois pas beaucoup à la distribution virtuelle?

Jean-François Denis - Pas à travers les sites généralistes... II y a cependant des exemples intéressants, dont celui du site www. notype.com consacré aux musiques électroniques et expérimentales. Ici aussi, pas d'objet matériel, de disque. Mais, à la différence de sites généralistes comme www.mp3.com, où tout est sur le même plan, dans ce cas-ci, le webmestre regroupe les musiques de son choix en albums virtuels qu'on peut télécharger gratuitement. II y a donc direction artistique, tout comme chez Empreintes DIGITALes. Cela facilite la tâche de l'auditeur.

Yves Daoust - Alors, pourquoi ne pas faire la même chose et éliminer l'étape de production matérielle?

Jean-François Denis - Parce que le mp3 n'offre pas encore la qualité sonore requise pour bien rendre l'électroacoustique (mais il faudra me reposer la question le jour où apparaîtra une nouvelle norme vraiment comparable au CD actuel...l, et aussi parce beaucoup de gens n'ont ni le temps et encore moins les moyens techniques de télécharger des fichiers de centaines de mégaoctets chacun.

Yves Daoust - Tu insistes beaucoup sur ton rôle de directeur artistique. Alors qu'estce qui motive tes choix? Quel est le profil du compositeur susceptible de se retrouver sur Empreintes DIGITALes?

Jean-François Denis - C'est une question délicate car le choix se fait de façon très... impulsive. Le travail du compositeur doit me plaire, mais il faut surtout que je ressente une... rigueur dans sa démarche. De plus, je dois sentir aussi la motivation du compositeur à poursuivre et à développer sa carrière, un élément important pour la diffusion du disque. Trop souvent un compositeur considère qu'une fois le CD produit, il peut passer à autre chose, alors qu'il faut encore faire vivre ces 
musiques, les présenter en concert et en parler lors d'entrevues à la radio et dans les médias écrits (imprimés ou électroniques).

Yves Daoust - J'aimerais revenir à la notion de créneau qu'on a abordée plus tôt. Tu disais que depuis quelques années tu as resserré le créneau d'Empreintes DIGITALes, te limitant aux musiques de support. Pourquoi?

Jean-François Denis - J'ai voulu m'assurer qu'il n'y ait pas d'ambiguïté quant au créneau d'Empreintes DIGITALes : on assiste depuis quelques années à un foisonnement important des musiques électroacoustiques qui éclatent dans tous les sens. C'est extraordinaire et porteur d'avenir. Mais je pense qu'il est crucial d'éviter la confusion des genres auprès des auditeurs. C'est pourquoi j'ai resserré l'offre, en gardant bien le cap sur les œuvres fixées. Mais afin de rendre compte de ce foisonnement et à la suite d'une rencontre avec David Turgeon, une des personnes derrière www.notype.com, i'ai fondé No Type, une nouvelle étiquette qui est le prolongement matériel du site web. C'est un nouveau couloir, spécialisé celvi-ci dans les musiques électroniques et expérimentales alternatives.

Yves Daoust - Mais n'y a-t-il pas risque de cloisonnement quand les corridors sont trop serrés? Où se loge un compositeur - et c'est de plus en plus fréquent chez les jeunes - qui pratique une sorte d'hybridation entre ce qu'on pourrait appeler l'électro "classique", issue de la tradition savante, et des approches plus alternatives, orales, issues souvent de la pop ou du techno?

Jean-François Denis - La musique expérimentale est toujours plus ou moins assise entre deux chaises. C'est au compositeur et à moi de décider du meilleur corridor pour tel ou tel projet de disque. Par ailleurs, sur le site de ma compagnie de distribution (www.electrocd.com), on y retrouve l'ensemble des titres mélangés, quelle que soit l'étiquette. Cela dit, pour en revenir à No type, une autre raison pour laquelle j'ai décidé de créer cette étiquette, c'est que le genre dit «électroacoustique" n'a pas toujours bonne presse. Il est souvent considéré par les gens des nouvelles musiques alternatives (et par bien d'autres!) comme académique (musique froide, stérile...). Plusieurs créateurs, dont le travail pourrait selon moi très bien entrer dans le grand genre électroacoustique, préfèrent être "associés " à la zone représentée par No Type. Eh bien! allons-y! II ne pourra y avoir que "contamination " (dans les deux sens!) : les amateurs de cette collection écouteront de l'électroacoustique (mais sans le savoir nommément), sans préjugé défavorable. Avec le temps, je pense que les cloisons se briseront, qu'il y aura contamination d'un genre à l'autre.

Yves Daoust - Donc, toujours ce clivage entre les genres...

Jean-François Denis - ... que beaucoup de journalistes entretiennent malheureusement, n'établissant pas de liens historiques entre les pratiques artistiques. Mais je n'ai pas voulu m'attaquer à ça, du moins pas directement. Je fais plutôt le pari que les habitués de No Type iront fureter dans l'étiquette voisine (Empreintes 
DIGITALes) et vice versa. II y a déjà des exemples: I'an dernier s'est tenu à Montréal un événement qui s'intitulait Dhomontellement. Fascinés par la musique de Francis Dhomont, de jeunes compositeurs d'électronique expérimentale ont "revisité » son œuvre...

Yves Daoust - Je reviens à cette prétendue mauvaise presse qu'aurait la musique électroacoustique. Un des problèmes de cette musique ne tientil pas au fait que, bien qu'étant une musique de support réalisée entièrement en studio, sa destination première est, règle générale, le concert? Ce sont des musiques pensées pour le concert, qui trouvent leur pleine signification dans ce cadre.

Jean-François Denis - II y a une différence fondamentale entre la musique instrumentale et la musique électroacoustique : cette dernière est une musique de médium. Elle est donc indissociable du support sur lequel elle est couchée, alors que l'enregistrement d'une musique instrumentale est essentiellement d'ordre documentaire.

Yves Daoust - D'accord, quand il s'agit de l'enregistrement d'un concert. Mais n'y a-t-il pas de plus en plus de compositeurs "instrumentaux", particulièrement chez les jeunes, qui pensent, au moins un peu, en fonction de l'enregistrement?

Jean-François Denis - II est vrai que le concert est devenu une forme très marginale de communication de la musique, particulièrement depuis le développement de la radio qui est devenue un fournisseur majeur de musique. La radio et le disque représentent certainement aujourd'hui plus de $90 \%$ de notre rapport à la musique.

Yves Daoust - Et cette écoute individuelle a complètement transformé notre rapport à la musique, à la forme, à la perception du temps. Le compositeur a perdu le contrôle...

Jean-François Denis - La musique est devenue un produit de consommation, donc les usages se sont multipliés.

Yves Daoust - Si les œuvres électroacoustiques sont des musiques de support, elles ne sont pas nécessairement pour autant des œuvres pour support dans la mesure où elles continuent d'être pensées et composées en fonction du concert et donc destinées à être écoutées dans une relation traditionnelle de représentation où la seule différence avec le concert instrumental réside dans le fait d'avoir remplacé les instruments par des enceintes acoustiques (on a même gardé le chef, qu'on appelle "diffuseur »...

Jean-François Denis - C'est sans doute là où le clivage entre les tendances est le plus manifeste. Le concert dans sa forme traditionnelle reste effectivement la situation idéale de diffusion pour la majorité des compositeurs électroacousticiens, le lieu où la musique prend tout son sens : le public est captif, silencieux, sous le contrôle du diffuseur. Mais comme le concert rejoint peu de monde, on voit dans 
la diffusion radiophonique et surtout dans l'édition discographique un moyen d'élargir le cercle des auditeurs, en espérant qu'une partie de ceux-ci, rejoints par ces médias, finira par venir au concert. Mais il y en a qui ont compris, et particulièrement chez les compositeurs des tendances alternatives des musiques dites électroniques, que "le médium, c'est le message", comme l'a dit Mcluhan. Ils composent donc pour le disque, ayant compris qu'une nouvelle relation musiquepublic a pris forme, particulièrement depuis la standardisation du support numérique. Is réalisent que le disque amène une relation à la forme, au temps, très différente des schèmes classiques des œuvres destinées d'abord au concert.

Yves Daoust - Mais qu'en estil alors du concert? Estil destiné à mourir?

Jean-François Denis - Paradoxalement, ces compositeurs inversent le processus des musiques non commerciales, si on peut dire : au lieu d'aller du concert au disque (comme en musique instrumentale contemporaine), ils reproduisent au concert l'approche qu'ils ont de l'œuvre-disque. Is réinventent ainsi le concert. Les œuvres sont ouvertes, le public va et vient comme il veut, parle, fume, boit... La présence des musiciens-interprètes est très discrète. Le concert devient (redevient) un espace social, un lieu d'échange, une forme de fête.

Yves Daoust - Est-ce que tu incites les compositeurs dits "savants» à développer davantage une conscience du médium "disque»?

Jean-François Denis - Non, je laisse les tendances suivre leur cours. Je préfère être un reflet de ce qui se passe plutôt qu'un moteur ; un miroir, légèrement déformant... II y a eu quand même les disques Électro clips (en 1990) et Miniatures concrètes (en 1998), qui étaient des disques concepts, et aussi, à venir, une œuvre pour disque, Le contrat, que j'ai commandée à Gilles Gobeil et René Lussier. Mais essentiellement mon rôle reste de rendre les musiques disponibles, accessibles, et de contribuer à la constitution d'un patrimoine. 


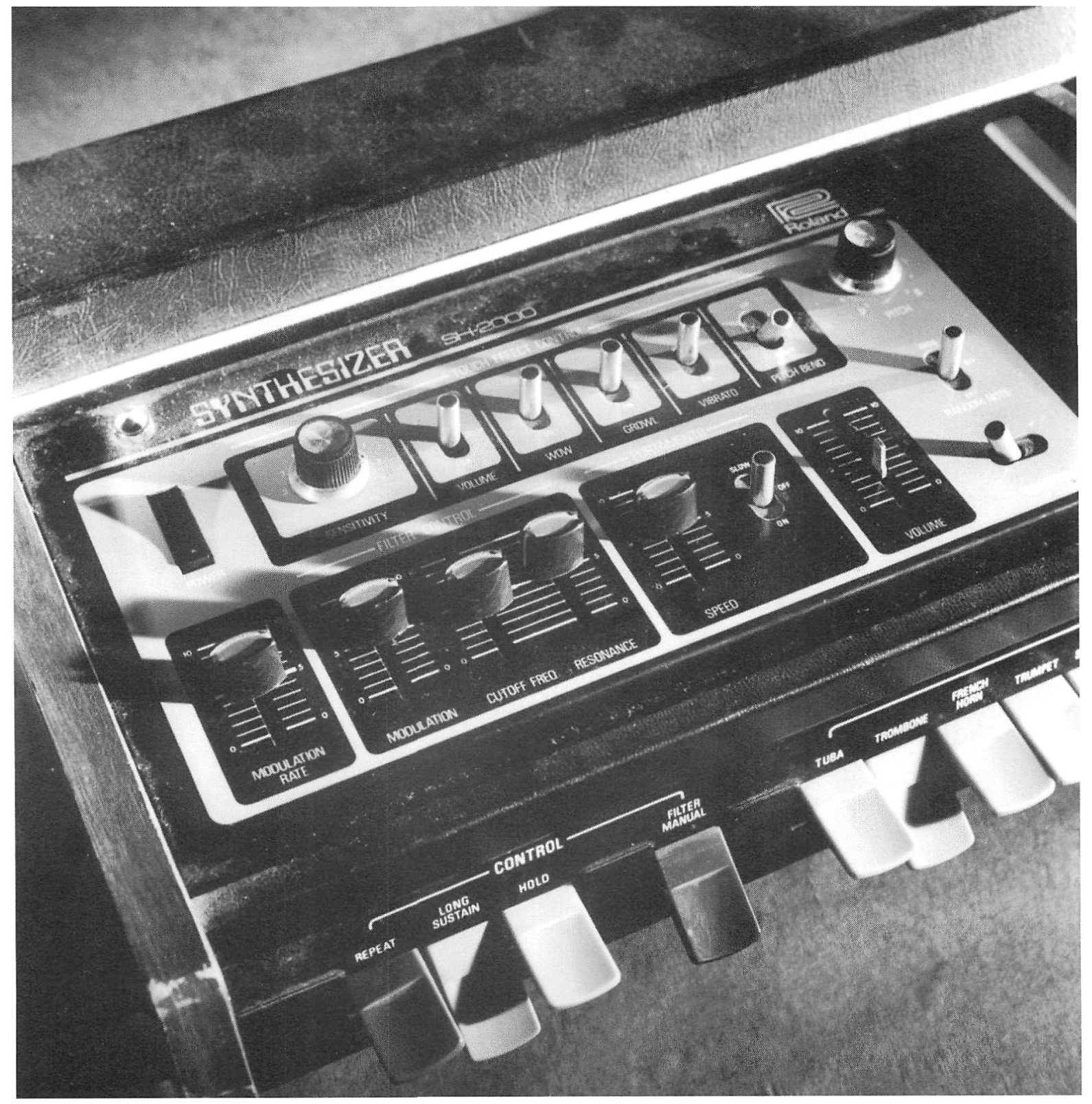




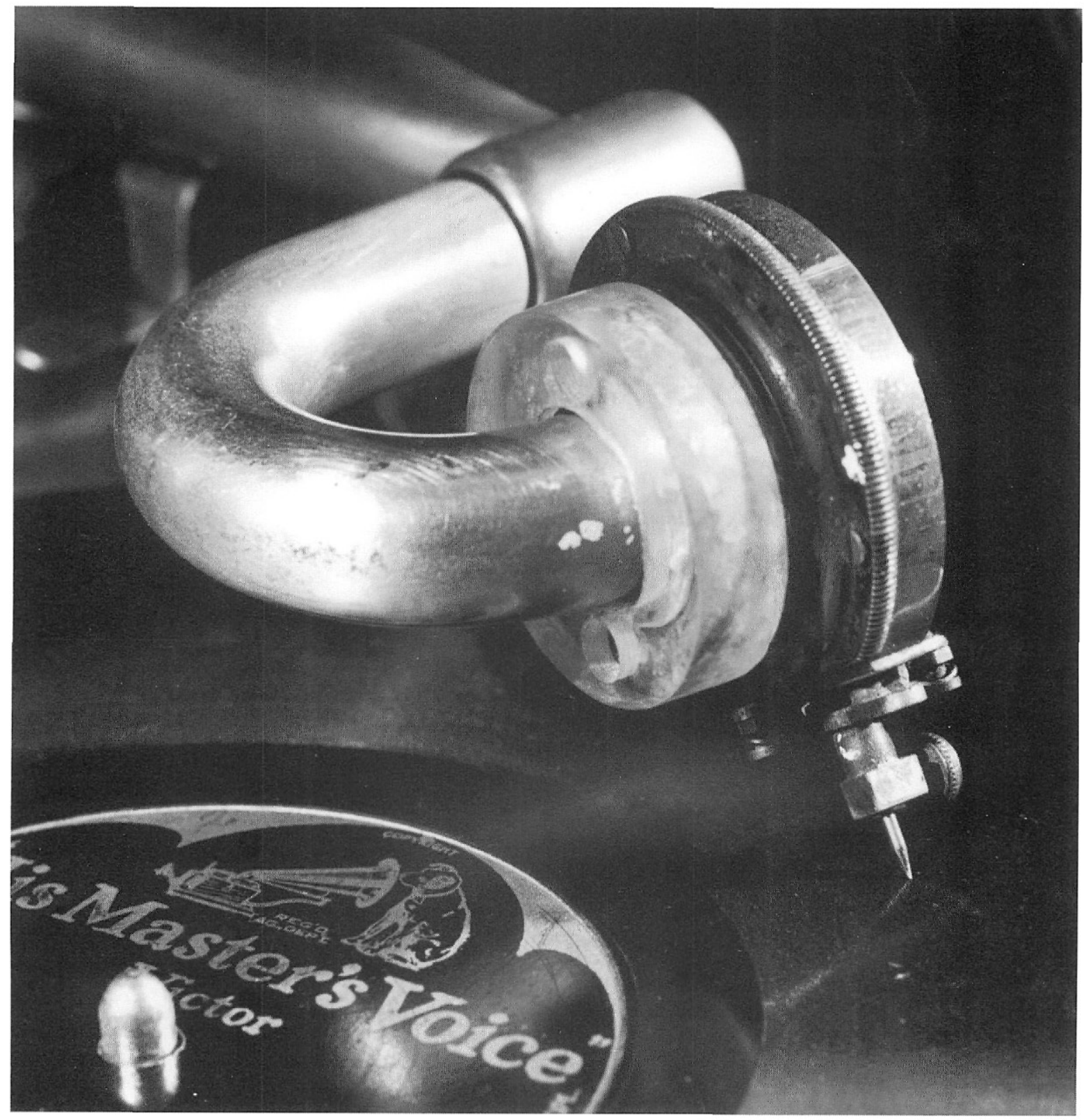

